\section{Dilemma moderner Medizin: Alloantikörper gegen Faktor VIII bei Hämophilie A häufig}

Eine öffentlich geförderte, randomisierte, unverblindete Studie mit Beteiligung weltweit verteilter Zentren ernüchtert und zeigt einmal mehr die Grenzen einer gezielten Substitutionstherapie auf: Mehr als 30\% der Patienten, die mit Faktor-VIII-Präparaten behandelt werden, entwickeln Alloantikörper.

$\mathrm{D}$ ie Daten von 251 Jungen unter 6 Jahren mit schwerer Hämophilie A, das heißt mit einer Faktor-VIII-Aktivität $<1$ IU/dl und ohne Nachweis von FaktorVIII-Alloantikörpern, wurden analysiert. Keines dieser Kinder hatte bislang Faktor-VIII-Präparate und maximal 5-mal ein Blutprodukt bekommen. Die eine Gruppe (125 Teilnehmer) erhielt nun aus menschlichem Blutplasma gewonnenen Faktor VIII, der auch Von-WillebrandFaktor enthielt. Knapp 27\% entwickelten Alloantikörper; 20 Jungen davon sogar hohe Antikörpertiter von $\geq 5$ Bethesda-
Einheiten. Die andere Gruppe (126 Teilnehmer) wurde mit rekombinanten Faktor-VIII-Präparaten versorgt. Hier kam es bei knapp $45 \%$ zur Entwicklung von Alloantikörpern; bei 30 Kindern fanden sich hohe Titer. Rechnerisch ergab sich eine um relativ $87 \%$ höhere Inzidenz von Alloantikörpern nach Substitution mit dem rekombinantem Präparat. Auch die ermittelte Hazard Rate bezüglich der Häufigkeit hochtitriger Alloantikörper sprach gegen den rekombinanten Faktor VIII. Die Berücksichtigung von Alter, Familienanamnese, Herkunftsland, Menge der erhaltenen Blutprodukte oder Produktklasse der rekombinanten Präparate führten zu keiner Ergebnisveränderung.

Peyvandi et al. A randomized trial of Factor VIII and neutralizing antibodies in Hemophilia A. N Engl J Med 2016;374:2054-64

\section{Kommentar}

Dieser, unter italienischer (mailändischer) Federführung stehender, sehr aufwendigen Studie zufolge, führen native humane Plasmafaktor-VIII-Produkte zu einer geringeren Immunreaktion als in Tierzellen angezüchtete rekombinante DNA-Produkte. In dieser Erkenntnis steckt Potenzial für ein besseres Verständnis der AlloantikörperEntstehung gegen verschiedene FaktorVIII-Präparate. Offensichtlich sind Genmutationen oder verbliebene Mengen an Faktor VIII im Plasma derzeit nicht beeinflussbare Risiken für die gefürchtete Antikörperentwicklung gegen Faktor VIII.

Dr. Thomas Hoppen

\section{Intravenöses Paracetamol ist bei Frühgeborenen wirksam}

In einer finnischen Studie wurde die Wirksamkeit von Paracetamol per Infusion an Frühgeborenen < 32 Schwangerschaftswochen untersucht. Der Verbrauch von Morphin ließ sich durch den Einsatz von Paracetamol signifikant senken.

A n 108 Frühgeborenen mit einem mittleren Geburtsalter von 28,4 Wochen und einem mittleren Geburtsgewicht von

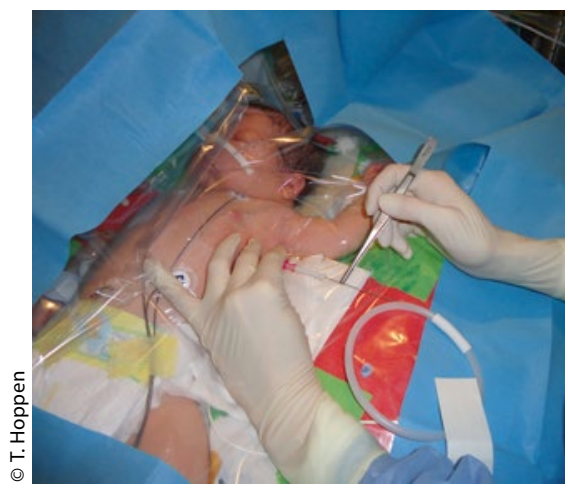

Die Anlage einer Pneumothoraxdrainage ist schmerzhaft.
$1.200 \mathrm{~g}$ wurde innerhalb der ersten $72 \mathrm{Le}-$ bensstunden Paracetamol (PCM) verabreicht. Die erste Dosis betrug $20 \mathrm{mg} / \mathrm{kg}$ $\mathrm{KG}$, es folgten $7,5 \mathrm{mg} / \mathrm{kg} \mathrm{KG}$ in sechstündigen Abständen. Eine entsprechende Vergleichsgruppe erhielt nur Morphin. Schmerzsymptome wurden anhand der „Neonatal Infant Acute Pain Assessment Scale" (NIAPAS) registriert. Es zeigte sich, dass Frühgeborene in der PCM-Gruppe signifikant weniger Morphin benötigten als die Patienten der Vergleichsgruppe. Bezüglich der Schmerzscores, Apnoen, Beatmungstage und unerwünschter Ereignisse einschließlich Lebertoxizität bestand kein Unterschied. Wenig überraschend war die PDA-Häufigkeit in der PCM-Gruppe signifikant geringer.
Härmä A et al. Intravenous paracetamol decreases requirements of morphine in very preterm infants. J Pediatr 2016;168:36-40

\section{Kommentar}

PCM ist als Schmerzmedikament für Frühgeborene effektiv. Im Editorial zu dieser Studie werden jedoch Aspekte der Patientensicherheit betont [Anker van der JN et al. J Pediatr 2016;168:13-4]. Der Stellenwert der Langzeitverträglichkeit, die neurokognitiven Verläufe und die Entwicklung von Asthma oder einer anderen atopischen Erkrankung bedürfen der systematischen Untersuchung bei Verwendung von PCM in einer derart vulnerablen Patientengruppe. Wünschenswert ist zudem nach ausgereizter nicht-pharmakologischer Schmerztherapie die ausschließliche Verwendung eines analgetischen Medikamentes bei Frühgeborenen und nicht einer Kombination. So lassen sich mögliche gefährliche Interaktionen untereinander bei Verwendung zweier oder weiterer Substanzen reduzieren. Übrigens ein Grundsatz, der in der Medizin generelle Bedeutung hat.

Dr. Thomas Hoppen 